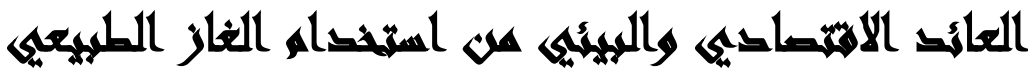

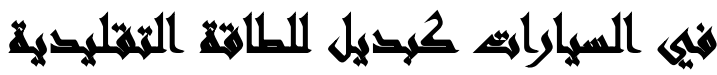

[17]

\author{
عبير فرحات علي(')- عمرو علي مصطفي توفيق(؟) - مي منصور فولي \\ ( ) كلية التجارة، جامعة عين شمس بات عل شركة انبي

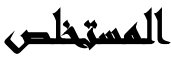

تهدف هذه الدراسة الي إيضاح اثر استخدام الغاز الطبيعي في السيارات كوقود بديل

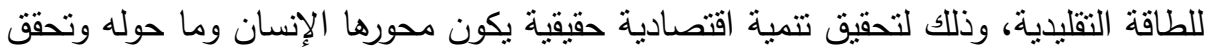

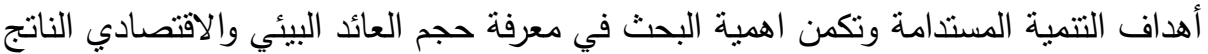

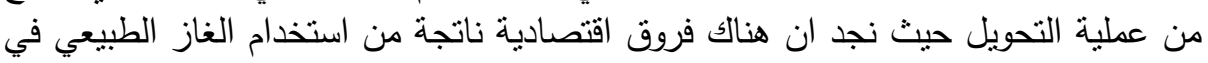

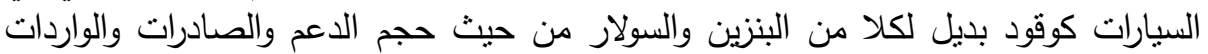

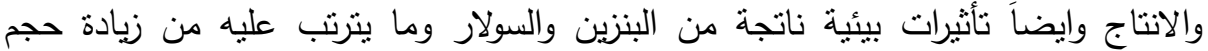

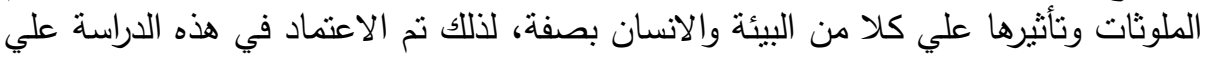

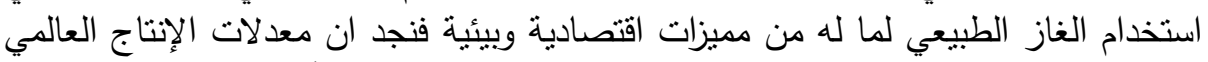

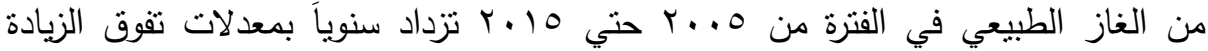

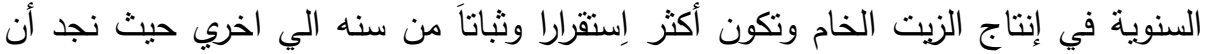

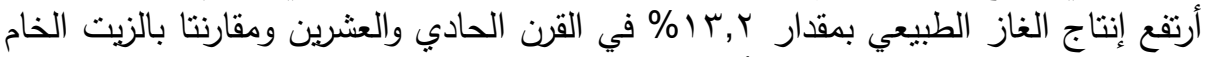

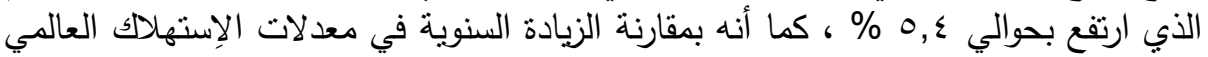

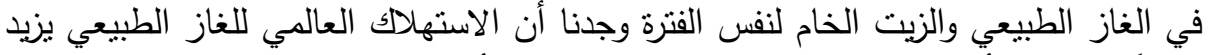

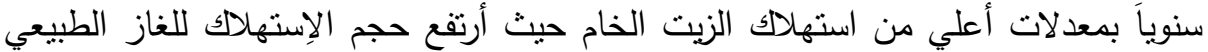

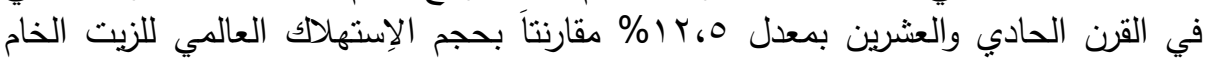

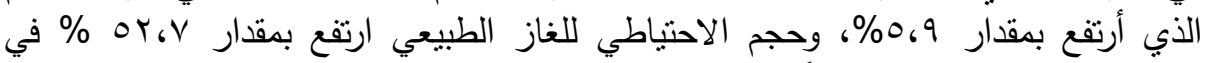

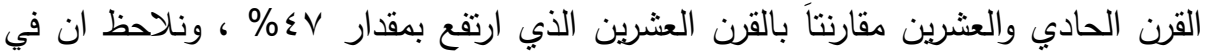

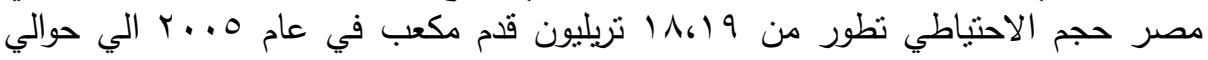
نV.99

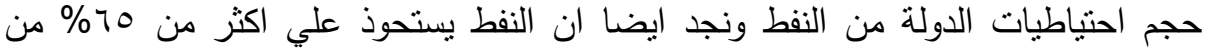

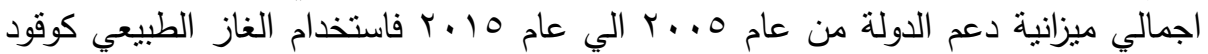

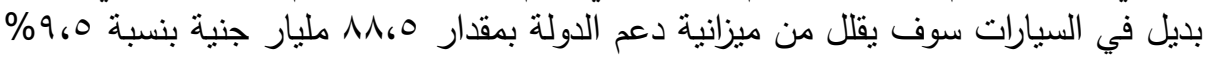

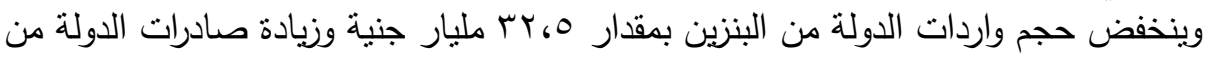


البنزين بمقدار r01،9 مليار جنية ، هذا بإضافة الي الاثار البيئية من استخدامه حيث

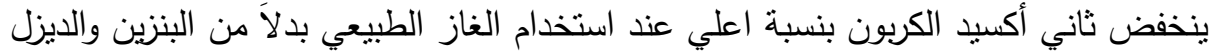

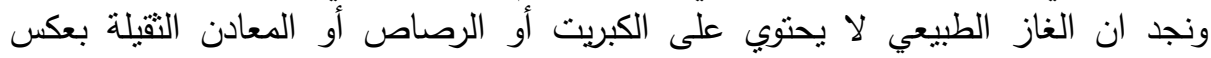

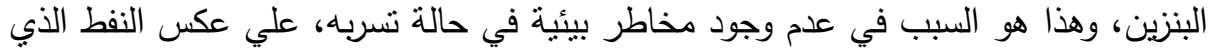
يتسبب في الآثار البيئية الضارة الناجمة عن تسرب الباب النفط.

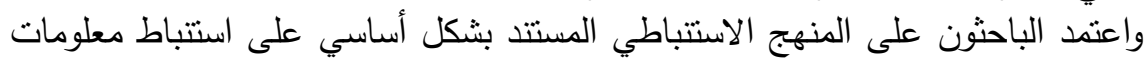

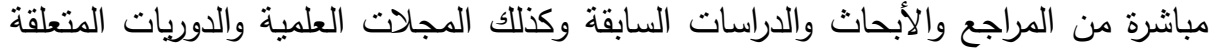

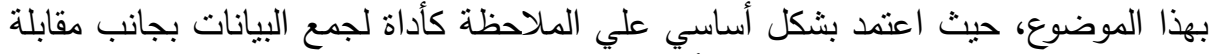
الجهات المعنيين بالدراسة وذللك في أطار مجتمع الدراسة وهو فئ فطاع البترول بما بمتلكه

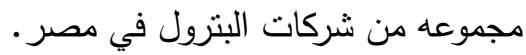
وتم الدراسة علي مجموعة من السيارات التي تستخدم الغاز الطبيعي والتي و صل عددها

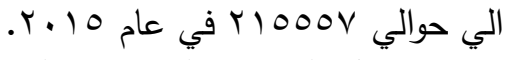

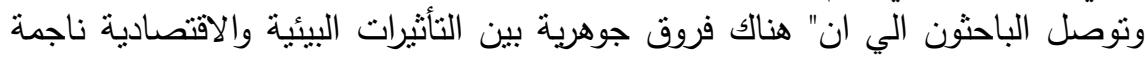

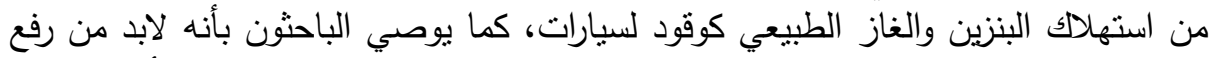

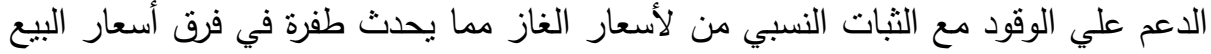

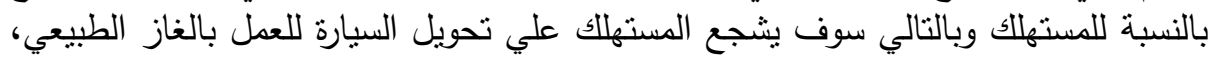

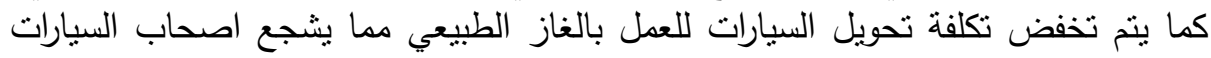

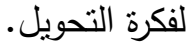

\section{ranodll}

مما لا شك فيه أن نوفير مصادر الطاقة المختلفة يعد من أهم التحديات اللازمة لدفع عجلة التتمية الاقتصادية الثاملة، ليس هذا فحسب وإنما هي ضرورية في كافة أنثكال الحياة

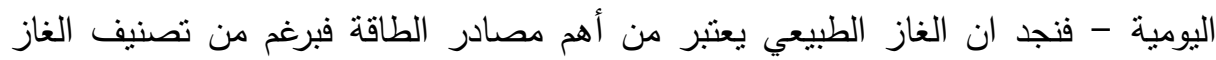

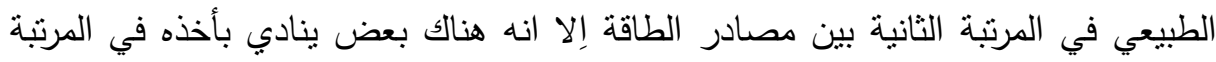
الاولي بين مصادر الطاقة المتجددة حيث انه يفوق علي البترول في ضئ فالكة مخلفاته الناتجة

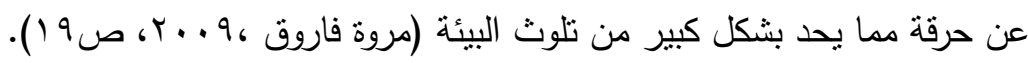
فبدون الطاقة تتوقف كافة الاشكال الحضارية في حياة الانسان وما كان الانسان ليستطيع أن يصل إلي ما وصل إلية الآن من تقدم تكنولوجي هائل أو ما يسمي بالثورة 
المعلومانية مروراً بالثورة الصناعية الاولي والثانية إلا من خلال إكتثافه لمصادر الطاقة

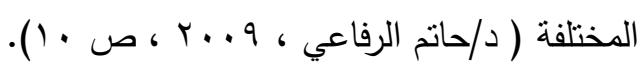

وتواجه مصر مثل العديد من الدول النامية الأخرى تحديات عديدة من بين تحديات اقتصادية واجتماعية وبيئية لتحسين مستوي معيثة أفرادها، وتوفير واستخدام الطاقة النظيفة هي أحد هذه التحديات سواء علي مستوي معيشة الأفراد أو علي المستوي الاقتصادي الهيكلي فئي

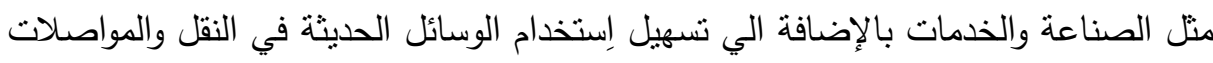

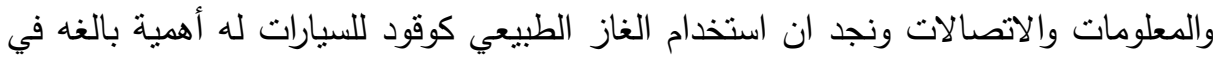
مصر نظراً لمزاياه البيئية والاقتصادية المتعددة ومن أهمها: تلافي الأثار الضارة للملوثات الناتجة عن الوقود السائل لإنخفاض العوادم الضارة الناتجة عن احتراقه لخلوه من الثوائب الثباه

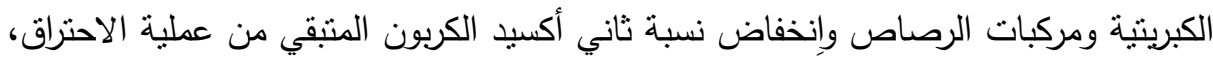

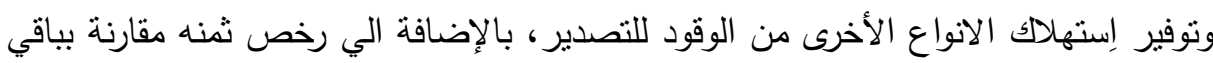

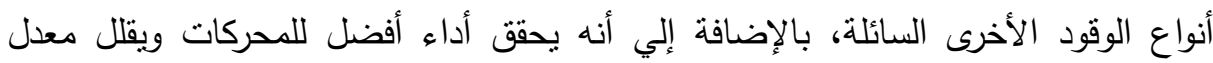

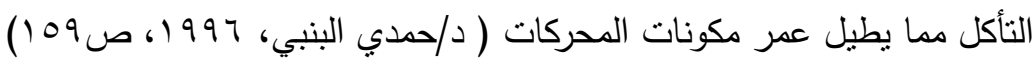
كما يؤدي إلي انخفاض معدلات تغيير زيوت التزييت لإنخفاض تكوين الرواسب، ومن اهم العوامل التي شجعت علي إِتخدام الغاز الطبيعي في وسائل النقل في مصر النتائج

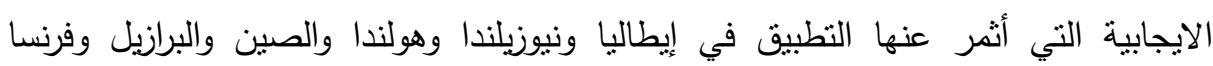
والمكسيك والهند وتايلاند وبنجلاديش ونيجيريا وباكستان وإيران وتركيا، فقامت مصر باختبار

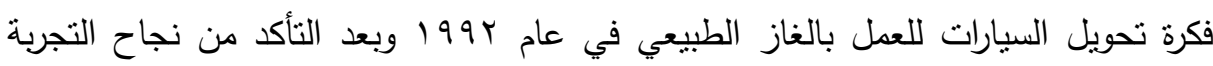
قامت وزارة البترول في عام 1997 بإنشاء شركتين متخصصنتين لإجراء عمليات تحويل

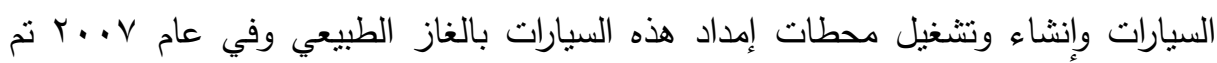
مشاركة القطاع الخاص في النهوض بصناعة الغاز الطبيعي وتم إنشاء العديد من محطات

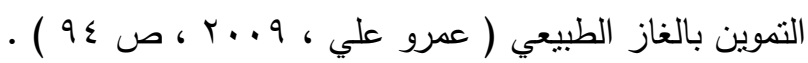




\section{التراسائ المربعية}

تضمنت الدراسة العديد من الدراسات المرجعية التي ناقشت وتناولت موضوع الدراسة وفيما يلي أهم هذه الدراسات مرتبة ترتيبا زمنيا:

1-اهتمت دراسة محمد النواوي r| r|: بدراسة الجدوى الاقتصادية البيئية للتحول من استخدام وقود السولار الي الغاز الطبيعي بمحركات الديزل وذللك عن طريق دراسة امكانية التغلب علي اسباب او تقليل تلوث الهواء المنمثل في عادم مركبات السيارات التي تستخدم وقود البنزين والسولار وذلك لتحقيق اقل معدلات نلوث بيئي والحصول علي هواء نظيف والتعرف علي امكانية تخفيف العب الاقتصادي علي موازنة الدولة وذلك باستخدام الغاز

$$
\text { الطبيعي بدلا من السولار }
$$

r-اهتمت دراسة أسامة الثيخ | | ـr: بعمل دراسة تحليلية للتكاليف البيئية والعائد منها في مجال الغازات البترولية وذلك عن طريق تحديد الانشطة البيئية وعناصر التكاليف المرتبطة بها ومحركات التكلفة لكل نشاط وإعداد الدراسة التطبيقية من الواقع العملي بسرد

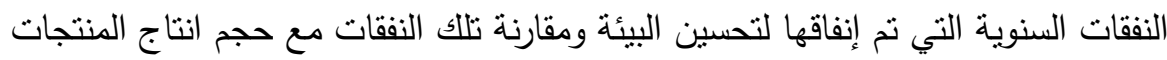
البترولية ومن اهم النتائج التي توصلت لها الدراسة ان حجم العائد يفوق علي النفقات حيث لم تتعدي النفقات المليون جنية مصري سنوياً وكانت العائدات المقدرة تخطت الأربعة

$$
\text { ملايين جنية سنوياً }
$$

r-اهتمت دراسة مروه الصادق 9 . . ب: بدراسة الاستثمارات الخاصة في قطاع الغاز الطبيعي وانعكاساتها علي التتمية في مصر وذلك عن طريق بحث ودراسة المتغيرات الداخلية والخارجية التي تؤثر علي الاستثمارات الأجنبية العاملة في صناعة الغاز الطبيعي في مصر والعمل علي إيجاد الحلول اللازمة لتحقيق مزيد من الاستشمارات واستخدام الغاز الطبيعي في العديد من القطاعات منل قطاع السيارات وتوصلت الدراسة الي انه لابد من من ماند إحلال الغاز الطبيعي محل المواد البترولية في الاستخدامات المحلية يمكن ان يسهم في تخفيض تكلفة الاحتياجات المحلية من الطاقة علي المدي الطويل. 
ع- اهتمت دراسة صبري البحيري V . . Y: بدراسة العائد الاقتصادي والبيئي لاستخدام الغاز

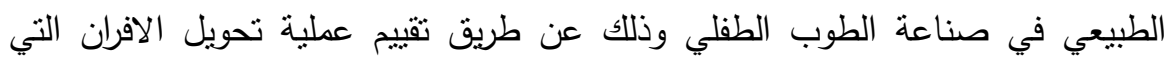

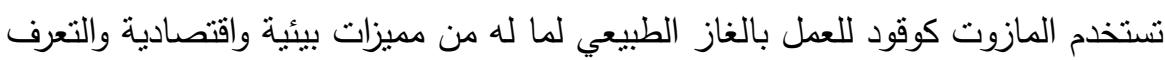
علي مميزات هذه العملية والوقوف علي أفضل الطرق التي يمكن اتباعها في مثل هذه لهن

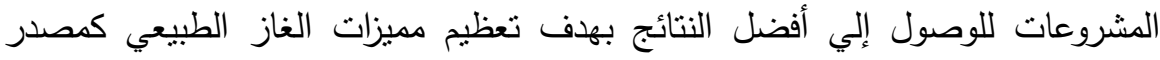
للحرارة في الافران الصناعية وخاصة صناعة الطوب الطفلي.

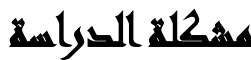

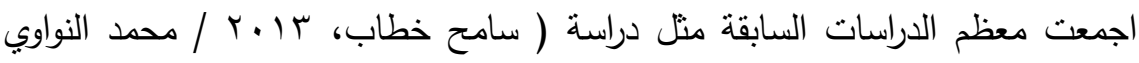

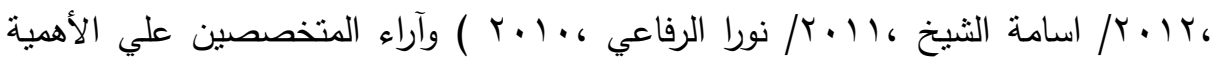
الاقتصادية والثأثثرات البيئية من استخدام الغاز الطبيعي كوقود بديل لكثير من الصناعات والقطاعات منل قطاع الكهرباء والمنازل وقطاع السيارات بصفه خاصة. حيث نجد ان البترول يغطي حوالي ب9\% من احتباجات الدولة لمصادر الطاقة الاولية

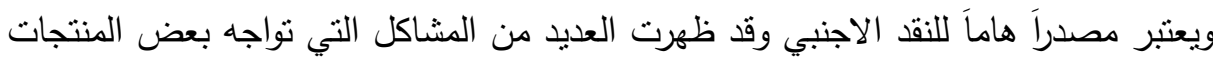

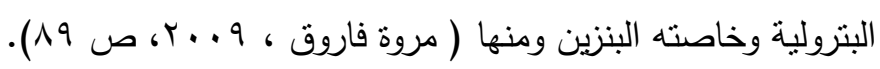
• مشكلات اقتصادية:

O انخفاض حجم الاتتاج للبنزين حيث يقدر بحوالي اگTV الف طن بنسبة •1\% من

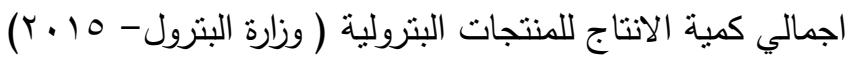

م ارتفاع حجم الواردات من البنزين حيث وصلت الي 709 الف طن بقيمة r.9 19 مليون

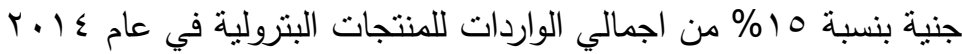
م ارتفاع تكلفة دعم الدولة للبنزين باعتباره المصدر الاساسي لوقود السيارات حيث وصل الي

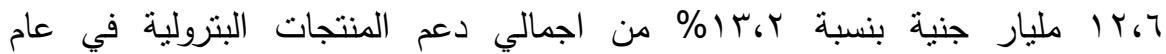

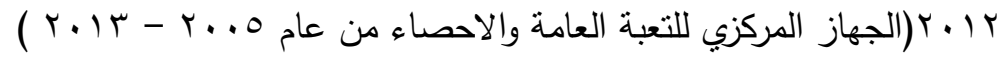


مشكلات بيئية:

O يشكل البنزين حوالي r.٪ من انبعاثات الكربون في مصر في حين ان الغاز الطبيعي

يشكل حوالي هب \% ( www.ngvjournal.com

م أن زيادة نسبة تركيزات ثاني أكسيد الكربون CO2 تؤدي إلى زيادة معدل التتفس، وعدم

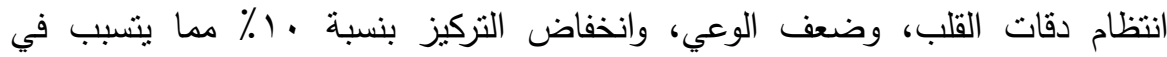

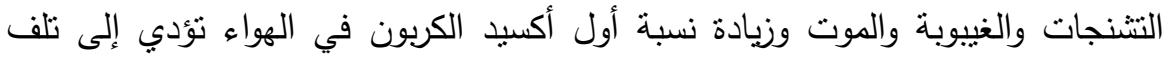

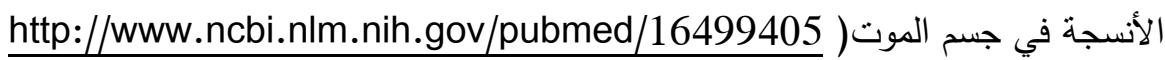

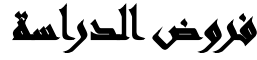

الفرضية الأولى: هناك عوائد اقتصادية من تحويل السيارات لاستخدام الغاز الطبيعي كوقود بديل. الفرضية الثانية: هناك فروف جوهرية ذات دلالة بين التأثيرات البيئية الناجمة من استهلاك البنزين والغاز الطبيعي كوقود للسيارات. الفرضية الثالثة: يمكن تعميم استخدام الغاز الطبيعي المضغوط كوقود بديل للبنزين في وسائل

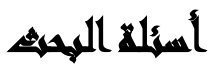

$$
\text { تسعي الاراسة الي الإجابة علي التساؤلات التالية: }
$$

• ما مدي وجود مزايا اقتصادية من استخدام الغاز الطبيعي في السيارات كوقود بديل للطاقة

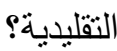
• ما مدي وجود مزايا بيئية من استخدام الغاز الطبيعي في السيارات كوقود بديل للطاقة 


\section{أهساهنم الصراسها}

تحاول تلك الاراسة تحقيق الأهداف الآتية: 1- دراسة العائد الاقتصادي لاستخدام الغاز الطبيعي في السيارات كوقود بديل للطاقة

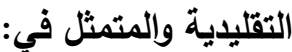

$$
\text { • تخفيف العبء علي موازنة الدولة في حجم الدعم المقدم علي بعض المنتجات البترولية. }
$$
• زيادة صادرات وخفض واردات مصر من بعض المنتجات البترولية.

• إلقاء الضوء علي حجم الاحتياطيات الكبيرة للغاز الطبيعي مما يشجع علي فكرة تحويل السيارات لاستخدام الغاز الطبيعي كوقود بديل.

r- دراسة العائد البيئي لاستخدام الغاز الطبيعي في السيارات كوقود بديل للطاقة التقليدية والمتمثل • التعرف علي سلبيات البنزين وايجابيات الغاز الطبيعي كوقود بديل للسيارات

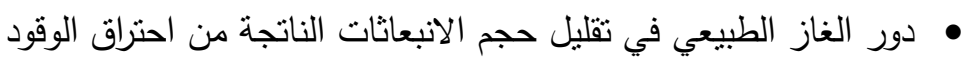

\section{مشوض القراسلة}

حدود زمنية: تتحصر فترة دراسة التجربة المصرية لاستخدام الغاز الطبيعي المضغوط

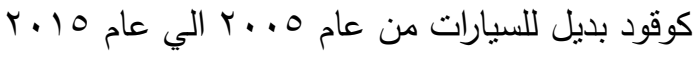
حدود مكانية: تشمل تطبيق الدراسة علي قطاع السيارات في مصر

\section{منهمج التوالها.}

منهج وصفي: تم من خلال تجميع البيانات والمعلومات التي ترتبط بالدراسة عن طريق الاطلاع على الكتب والدوريات والنقارير والأبحاث والدراسات المنشورة، وأيضاً مطبوعات

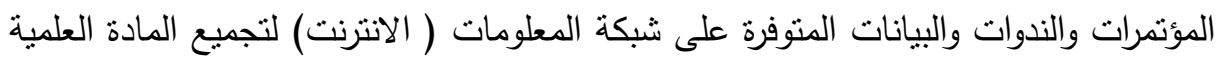
والبيانات المتاحة عن البحث محل الدراسة. 
منهج استتباطي: حيث تم استخدام التحليل الاستتباطي من حيث استخلاص النتائج وتحليلها،

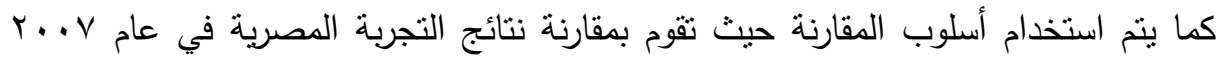
وما تم الوصول الية في عام 10 ـ r.

\section{أهمر السواسم}

الناحية العلمية: إلقاء الضوء علي الاضرار البيئية الناجمة من استخدام البنزين كوقود للسيارات والمميزات الاقتصادية التي تعود علي الدولة من استخدام الغاز الطبيعي من حيث انخفاض نسبة الدعم المقدم علي البنزين وزيادة الصادرات وتقليل الواردات من البنزين وزيادة

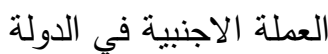
الناحية العملية: تقييم التجربة المصرية لاستخدام الغاز الطبيعي المضغوط كوقود بديل في

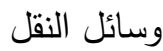

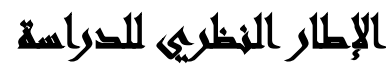

- العائد الاقتصادي: هو العائد او المنفعة التي تعود علي الدولة او الفرد من استخدام الطاقة النظيفة مما يؤدي الي تقليل الانبعاثات الضارة في الهواء و توفير تكلفة الدواء التي لهودي تتحملها الدولة والاقراد - العائد البيئي: هي الاثار البيئية الناتجة سواء كانت علي البيئة او الانسان من استخدام

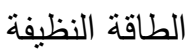
- تلوث الهواء: هو إحداث تغير في البيئية الهوائية التي تحيط بالكائنات الحية بفعل الانسان وأنشطته اليومية مما يؤدي الى ظهور بعض المواد التي نتلاءم مع المكان الذي يعيش فيه

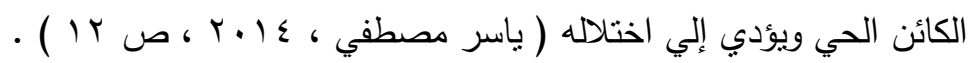




\section{أولاً: تعريف الغاز الطبيعي:}

- الغاز الطبيعي أحد مصادر الطاقة البديلة عن النفط وهو من المحروقات عالية الكفاءة قليلة التكلفة والانبعاثات الملوثة للبيئة وهو أهم مورد للطاقة في الصناعات الكيميائية

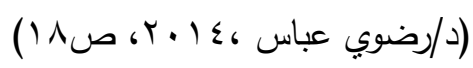

- ميتكون الغاز الطبيعي " من غاز الميثان CH4 " وهو غاز ( أو مركب ) مع ذرة كربون

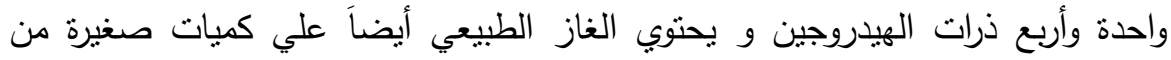
السوائل الهيدروكربونية والغازات غير الهيدروكربونية، والغاز الطبيعي يمكن استخدامه

$$
\text { كوقود أو في المواد الكيميائية (www.eia.gov) }
$$

ثانياً: إنتاج الغاز الطبيعي في مصر يتم انتاج الغاز الطبيعي من ثلاثة حقول رئيسية • حقل ابو ماضي: ويقع هذا الحقل في الثمال الثرقي من الدلتا ويتم نقل الغاز داخل انابيب إلي مواطن الاستهلاك وأهمها مصنع الأسمدة ومحطات نوليد الكهباء وشركات

$$
\text { الغزال والنسيج }
$$

• حقل ابو الغراديق: يقع هذا الحقل في الصحراء الغربية علي بعد •و كم من القاهرة ويرتبط هذا الحقل بمناطق الاستهلاك بخط أنابيب تصله بمنطقة دهشور ويغذي منطقة

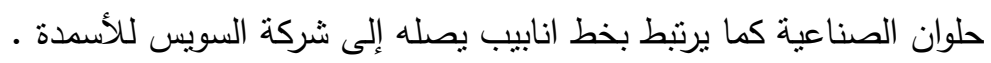

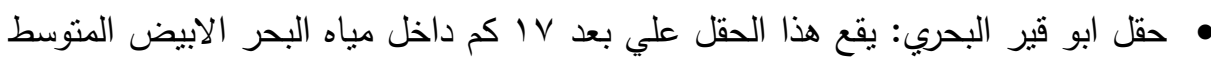

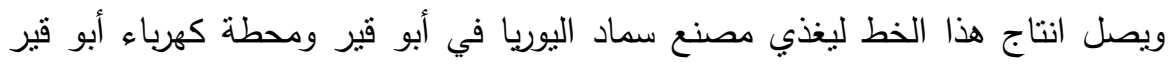

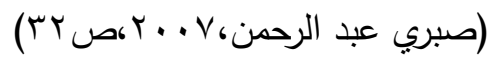

ثالثاً: الفرق بين النفط الخام والمنتجات البترولية: النفط الخام هو خليط من الهيدروكربونات التي شكلت من النباتات والحيوانات التي عاثت قبل ملايين السنين. النفط الخام هو الوقود الأحفوري، وكان موجودا في شكل سائل في برك تحت الأرض أو الخزانات، في المساحات الصغيرة داخل الصخور الرسوبية، وبالقرب من السطح في القطران (أو الزيت) الرمال، وبعد إزالة النفط الخام من الأرض يتم إرساله إلى مصفاة حيث يتم فصل أجزاء مختلفة

$$
\text { المجلد السادس والثثلاثون، الجزء الثالث، ديسمبر r } 1 \text { ـ ب }
$$


من النفط الخام الى منتجات بترولية قابلة للاستعمال. وتتشل هذه المنتجات النفطية البنزين، نواتج التقطير مثل وقود الديزل وزيت التدفئة ووقود الطائرات والبتروكيماويات من المواد الخام، والثمع، وزيوت التشحيم، والأسفلت ، بينما البنزين هو الوقود المصنوع من النفط الخام وغيرها www.eia.gov () من السوائل النفية، ونية

\section{الجراعايت الصواسمة}

- قامت هذه الدراسة بالاعتماد علي مجموعة من الكتب المتخصصة بموضوع الدراسة منل "

البترول بين النظرية والتطبيق - البترول المصري تجارب الماضي وآفاق المستقبل -

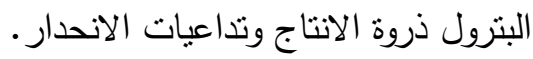

- تحليل التقارير المرتبطة بموضوع الدراسة وهذه التقارير التي تصدر من مجموعة جهات

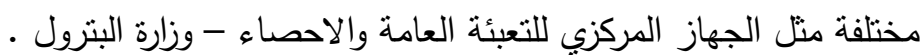

- www.eia.gov تحليل البيانات والمؤشرات من مواقع الكترونية مختلفة مثل www.ngvjournal.com

- ربط التقارير والمؤشرات والبيانات بموضوع الدراسة " العائد الاقتصادي والبيئي من استخدام الغاز الطبيعي في السيارات كبديل للطاقة التقليدية " ومدي نطبيق ونجاح هذه ولهن

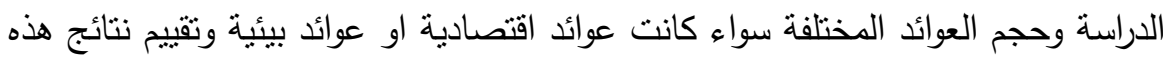
الدراسة . - تقوم الدراسة بتحليل البيانات والمؤشرات خلال الفترة من عام ه. . ب - 10 ـ ؟. 


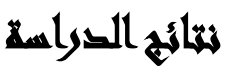

\begin{tabular}{|c|c|}
\hline \multicolumn{2}{|l|}{ التأثير الاقتصادي } \\
\hline تتخفض ميزانية دعم الدولة بمقدار N1،0 مليار جنية & دعم الدولة \\
\hline تتخفض بمقدار مr.0 مليار جنية & 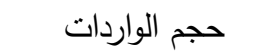 \\
\hline ارتفعت بمقدار rوه r مليار جنية & حجم الصادرات \\
\hline ارتفعت خلال الفترة بمقدار 54\% & محطات التموين \\
\hline ازدادت بمقدار Yr) & مراكز التحويل \\
\hline 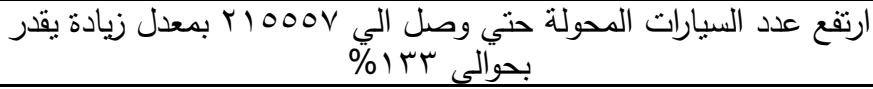 & عدد السيارات التي تم تحويلها \\
\hline \multicolumn{2}{|l|}{ التأثنر البيئي } \\
\hline في حالة السرعة ينخفض بمتوسط حوالي 01، (01\% & \multirow{2}{*}{$\begin{array}{l}\text { اكسيد الكربون } \mathrm{Cl}>2000 \\
\mathrm{CO}_{2}\end{array}$} \\
\hline 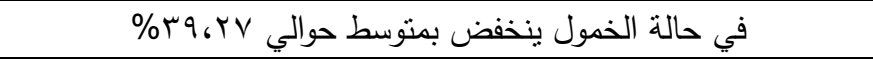 & \\
\hline في حالة السرعة ينخفض بمتوسط حوالي 0V، \% & \multirow{2}{*}{$\begin{array}{c}\text { Cc }=1400 \\
\text { hc الهيدروكربونات }\end{array}$} \\
\hline 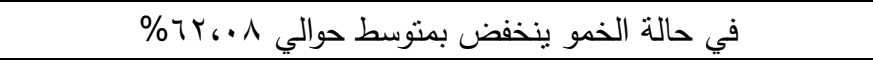 & \\
\hline في حالة السرعة ينخفض بمتوسط حوالي A1، ،91\% & \multirow{2}{*}{$\begin{array}{l}\text { الكربون Cc =1200 } \\
\text { Co اول co }\end{array}$} \\
\hline في حالة الخمول ينخفض بمتوسط حوالي اس،9Y\% & \\
\hline
\end{tabular}

- ثبتت صحة الفرضية الاولي والتي تتص علي " هناك عوائد اقتصادية من تحويل السيارات لاستخدام الغاز الطبيعي كوقود بديل وذلك من خلال:

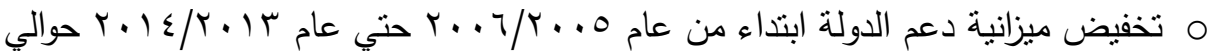

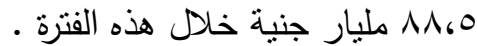

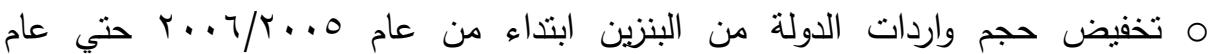

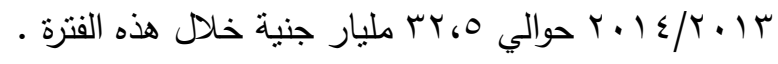

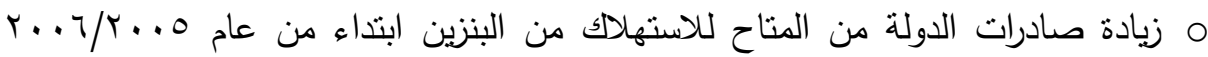

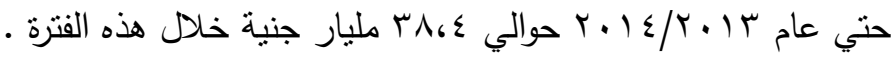

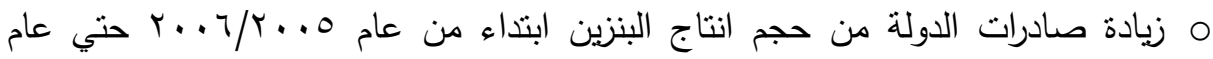

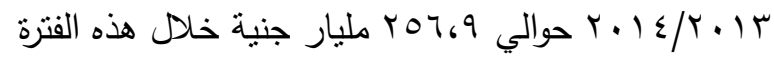




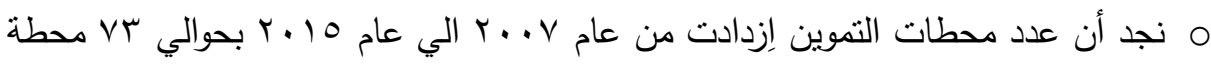

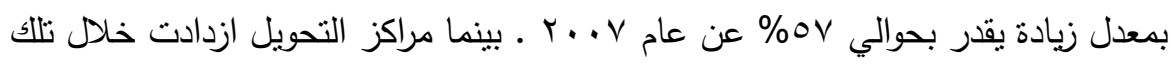

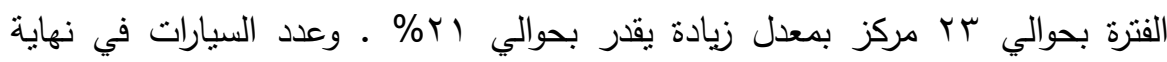

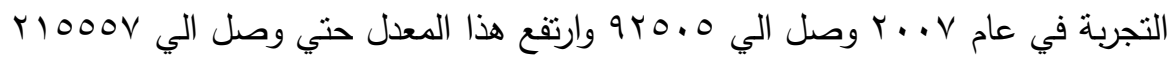

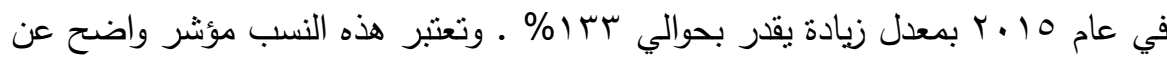

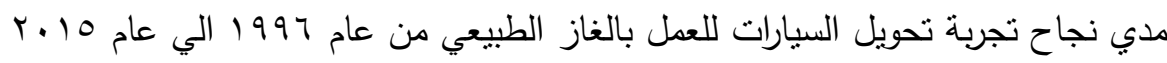

- ثبتت صحة الفرضية الثانية والتي تنص علي " هناك فروق جوهرية بين التأثيرات البيئية الناجمة من استهلاك البنزين والنغاز الطبيعي كوقود لسيارات

م حيث ان عملية تحويل السبارات للعمل بالغاز الطبيعي لها تأثثر إيجابي علي البيئة وتقليل حجم الانبعاثات الناتجة من عوادم السيارات فعلي سبيل المثال: ينخفض ثناني اكسيد

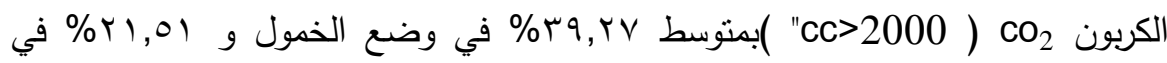

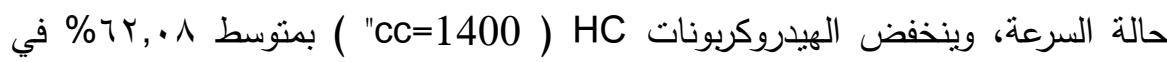

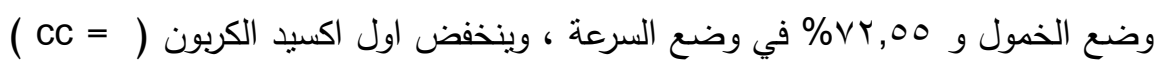

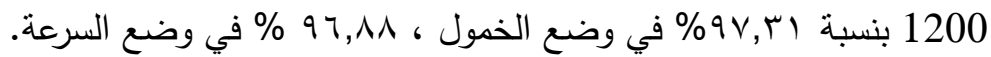
- ثبتت صحة الفرضية الثالثة والتي تتص علي " يمكن تعميم استخدام الغاز الطبيعي المضغوط كوقود بديل للبنزين في وسائل النقل "

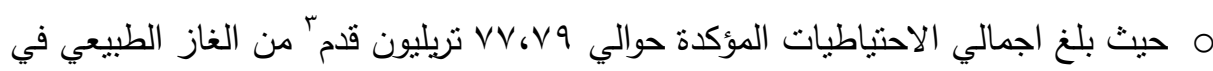
عام 10 10 مما بضمن بشكل كافي ان يتم استخدام الغاز الطبيعي كوقود للسيارات ليس هذا فقط بل يمكن ان ينم استخدامه في جميع الصناعات ويصبح المصدر الاول للطاقة 


\section{تموسياه الصراسمة}

نستطيع في ضوء النتائج السابقة ان نقترح عدداً من التوصيات والتي قد تكون مفيدة في تطبيق استخدام الغاز الطبيعي كوقود بديل للسيارات مما يؤنثر بالإيجاب علي الأداء الاقتصادي ويمكن تقسيم هذه التوصيات الي توصيات قصيرة الاجل وتوصيات طويلة الاجل: توصيات قصيرة الأجل: م التوسع في شبكات الغاز الطبيعي وعمل دراسة جدوي جغرافية أكثر دقة عند إنشاء محطات لتغطية مواقع جديدة مما يعني زيادة حجم السوق، وإمكانية انتشار المحطات

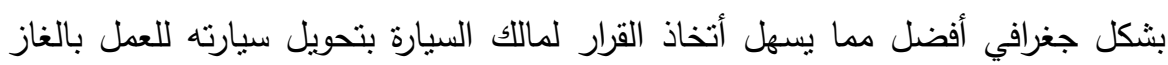

$$
\text { الطبيعي. }
$$

م استخدام الضرائب ورسوم التراخيص للسيارات التي تعمل بالغاز كأدوات تحفيزية (تخفيض

$$
\text { الضرائب ورسوم التراخيص) }
$$

م رفع الدعم علي الوقود ومع الثبات النسبي لأسعار الغاز مما يحدث طفرة في فرق أسعار البيع بالنسبة للمستهلك وبالتالي سوف يشجع المستهلك علي تحويل السيارة للعمل بالغاز

$$
\text { • الطبيعي }
$$

م خفض تكلفة تحويل السيارات للعمل بالغاز الطبيعي مما يشجع اصحاب السيارات لفكرة

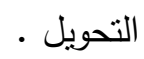

م التوعية البيئية المستمرة من أضرار البنزين علي البيئة والصحة والمميزات الاقتصادية

$$
\text { وغير الاقتصادية للغاز الطبيعي بالنسبة لصاحب السيارة . }
$$

\section{توصيات طويلة الأجل:}

م إنثاء وكالة متخصصة في إدارة سوق الغاز الطبيعي (CNG ) وتكون حلقة وصل حقيقية بين أطراف السوق بما فيها المستهلك، وتقوم هذه الادارة علي حل المشكلات الفنية

ومشاكل التموين وتكلفة التحويل وما يستجد من مشاكل.

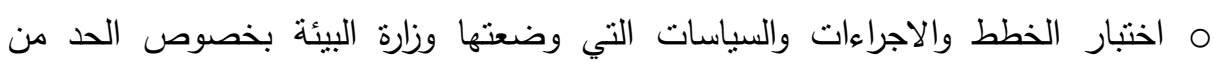

التلوث الناتج من المركبات للتأكد من نجاحها.

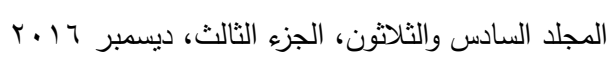


O استخدام التكنولوجيا في تطوير حجم اسطوانات الغاز الطبيعي للقضاء علي مشكلة كبر حجم الاسطوانات في سيارات الغاز الطبيعي.

م استيراد السيارات التي تعمل بالغاز الطبيعي وزيادة حجم السوق بهذا النوع من السيارات.

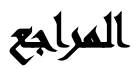

الجهاز المركزي للتعبة العامة والاحصاء - حركة الانتاج والتجارة الخارجية والمتاح من من

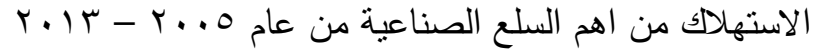

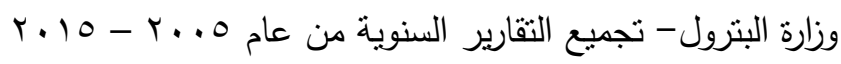

حاتم الرفاعي(9 + . †): البترول ذروة الانتاج وتداعيات الانحدار - كلية هندسة - جامعة عين

حمدي البنبي(999 (1)): البترول المصري تجارب الماضي وآفاق المستقبل - دار المعارف حمدي البنبي(799 (1): البترول بين النظرية والنطبيق - دار المعارف رضوي عباس السلاموني(ع ( ب): قسم تطوير العمليات - معهد بحوث البترول - مجلة البترول والعلوم البيئية - العدد السادس يونيو البعائ

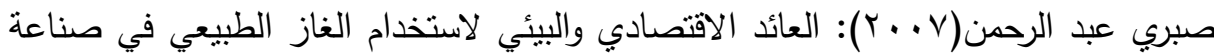

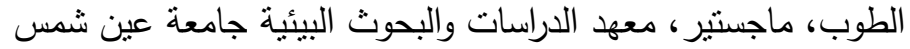

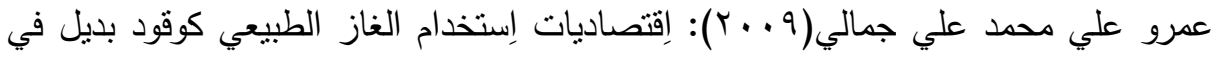
وسائل النقل - ماجستير - معهد الدراسات والبحوث البيئية- جامعة عين شمس لفي مروة فاروق محمد الصادق(9 . . ب): الاستثمارات الخاصة في قطاع الغاز الطبيعي وانعكاسها

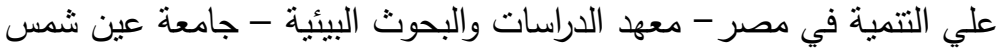
ياسر مصطفي: التلوث البترولي وطرق المعالجة - مجلة البترول والعلوم البيئية - العدد

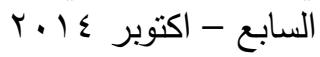

http://www.eia.gov/energyexplained/index.cfm?page=natural_gas_hom e $09 / 10 / 2016$

http://www.ngvjournal.com/50-reasons-for-using-ngveng/8/11/2016 01:10 am 
عبير فرحات علي وآخرون

\title{
THE ECONOMIC AND ENVIRONMENTAL BENEFIT FROM OF USING NATURAL GAS IN CARS AS AN ALTERNATIVE TO TRADITIONAL ENERGY
}

\author{
Ali, Abeer, F. ${ }^{(1)}$; Tawfik, A. A. M. ${ }^{(2)}$ and Fouly, M. M. \\ 1) Faculty of Commerce, Ain Shams University 2) Enppi Company
}

\begin{abstract}
This study aims to clarify the effect of the use of natural gas in the car as a fuel alternative to traditional energy, so as to achieve real economic development, human-centered and around and achieve sustainable development objectives, lies the importance of research to find out the size of the environmental and economic returns resulting from the conversion process, where we find that there are economic differences resulting from the use of natural gas in the automotive alternative for both gasoline and diesel fuel in terms of the size of the support, exports and imports, production and also environmental impacts resulting from the gasoline and diesel fuel and the consequent increase in the volume of pollutants and their impact on both the environment in general and man in particular, so I was relying on this the study on the use of natural gas because it is considered as the most important kind of Fossil fuels and one of the alternative resources of power instead of the oil and it is one of the fuels with high efficiency low cost and low polluting emissions and it is an important resource for the power in chemical industries as it has many environmental and economic advantages, We find that the international production rates of natural Gas in the period between 2005 and 2015 were with annual rates which surpass the annual increase in the rates of crude oil production and they are more stable and consistent from a year to another year where we find that the production of the natural gas were increased with rate $13.2 \%$ in the twenty-first century in comparison with the crude oil production which was increased about $5.42 \%$. By

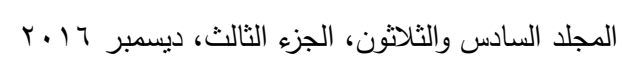


comparing the annual increase in the international rate of the natural gas consumption and the crude oil in the same period we found that the international consumption is being increased annually with rates which are higher than the rates of the crude oil consumption where the consumption of the natural gas is increased in the twenty-first century with rate $12.5 \%$ in comparison with the international consumption for the crude oil which is increased to $5.9 \%$. the residuals of the natural gas was increased to be about $52.7 \%$ more in the twenty-first century than the twenties century which was increased to be about $47 \%$, we notice that, in Egypt, the reserves of the natural gas was increased from 18.19 trillion cubic feet in 2005 to around 77.99 trillion cubic feet in 2015 which made its share of the fossil fuels reserves is more than 50 $\%$ from the country reserves of the fossil fuel and we find that the fuel consumes $65 \%$ the support budget of the country share in 2005 till 2015. The use of the natural gas as an alternative fuel in cars will decrease the support budget of the country to about 88.5 billion pounds with a portion about $9.5 \%$ and reduces the state imports of petrol with about 32.5 billion pounds and the increase of the state exports of petrol with about 256.9 billion pounds. in addition to the environmental outcomes behind its use where the portion of the Carbon Dioxide becomes less by the use of the natural gas instead of the petrol and diesel and carbon dioxide contributes in changing the international climate because of the phenomenon of the global warming and we find that the natural gas did not contain sulfur, lead, particles, or the heavy metals in comparison with petrol and it is a non-toxic gas and did not pollute the water at all; and this is the reason behind that there are no environmental hazards in the case of the its leak, on the contrary to the crude oil which results in a great harm effects on the environment by the oil leak.

The researcher depends on deductive approach is primarily based on the development of information directly from the references and research and previous studies as well as scientific journals and periodicals on the subject and The researcher depends on observation as a tool for collecting data next to interview those involved in the study 
عبير فرحات علي وآخرون

and in the framework of the study population, which is petroleum sector, as represented by a total of of the oil companies in Egypt .

The researcher found that "there are significant differences between the environmental and economic impacts arising from the gasoline and natural gas consumption as a fuel for cars, as the researcher recommends that there must be the removal of subsidies on fuel with the relative stability of prices for gas than a boom in sales prices squads for the consumer and thus will encourage the consumer happens Ali car conversion to natural gas, are also reduced the cost of converting cars to natural gas, which encourages car owners to the idea of conversion. 